Biocompatibility of nanostructured boron doped diamond for the attachment and proliferation of human neural stem cells

This content has been downloaded from IOPscience. Please scroll down to see the full text. 2015 J. Neural Eng. 12066016

(http://iopscience.iop.org/1741-2552/12/6/066016)

View the table of contents for this issue, or go to the journal homepage for more

Download details:

IP Address: 128.41.35.28

This content was downloaded on 01/04/2016 at 15:39

Please note that terms and conditions apply. 


\title{
Biocompatibility of nanostructured boron doped diamond for the attachment and proliferation of human neural stem cells
}

\author{
Alice C Taylor ${ }^{1}$, Barbora Vagaska ${ }^{2}$, Robert Edgington ${ }^{1}$, Clément Hébert ${ }^{3}$, \\ Patrizia Ferretti ${ }^{2}$, Philippe Bergonzo ${ }^{3}$ and Richard B Jackman ${ }^{1}$ \\ ${ }^{1}$ London Centre for Nanotechnology and Department of Electronic and Electrical and Engineering, \\ University College London, 17-19 Gordon Street, London, WC1H 0AH, UK \\ ${ }^{2}$ Institute of Child Health, University College London, 30 Guilford Street, London, WC1N 1EH, UK \\ ${ }^{3}$ CEA-LIST, Diamond Sensors Laboratory, Gif-sur-Yvette F-91191, France
}

E-mail: r.jackman@ucl.ac.uk

Received 8 April 2015, revised 22 July 2015

Accepted for publication 26 August 2015

Published 19 October 2015

\begin{abstract}
Objective. We quantitatively investigate the biocompatibility of chemical vapour deposited (CVD) nanocrystalline diamond (NCD) after the inclusion of boron, with and without nanostructuring. The nanostructuring method involves a novel approach of growing NCD over carbon nanotubes (CNTs) that act as a 3D scaffold. This nanostructuring of BNCD leads to a material with increased capacitance, and this along with wide electrochemical window makes BNCD an ideal material for neural interface applications, and thus it is essential that their biocompatibility is investigated. Approach. Biocompatibility was assessed by observing the interaction of human neural stem cells (hNSCs) with a variety of NCD substrates including un-doped ones, and NCD doped with boron, which are both planar, and nanostructured. hNSCs were chosen due to their sensitivity, and various methods including cell population and confluency were used to quantify biocompatibility. Main results. Boron inclusion into NCD film was shown to have no observable effect on hNSC attachment, proliferation and viability. Furthermore, the biocompatibility of nanostructured boron-doped NCD is increased upon nanostructuring, potentially due to the increased surface area. Significance. Diamond is an attractive material for supporting the attachment and development of cells as it can show exceptional biocompatibility. When boron is used as a dopant within diamond it becomes a p-type semiconductor, and at high concentrations the diamond becomes quasi-metallic, offering the prospect of a direct electrical device-cell interfacing system.
\end{abstract}

Keywords: stem cells, diamond, biocompatibility

\section{Introduction}

Diamond is a material with extreme physical, chemical and electrical properties [1], it is inert [2], robust and has high longevity. Nanocrystalline diamond (NCD) films produced by chemical vapour deposition (CVD) display very similar

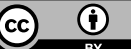

Content from this work may be used under the terms of the Creative Commons Attribution 3.0 licence. Any further distribution of this work must maintain attribution to the author(s) and the title of the work, journal citation and DOI. properties [3]. The passive chemical nature and the desirable tuneable electrical properties of diamond, including its wide electrochemical window, make it a highly desirable material for many bio-applications, specifically for interfacing with electrical cells as electrodes. The main hindrance of using planar films of diamond as an electrode material is the relatively low capacitance values obtained; an ideal electrode material has a high capacitance and this can be increased in diamond by increasing the surface area of the electrode. This has been achieved via seeding nanodiamonds on carbon nanotubes (CNTs) [4] and thus nanostructuring the diamond 
films, such films have shown excellent electrical properties. Here, these substrates have been investigated to see if the inclusion of boron and the effect of increased surface area via the nanostructuring has any observable outcome on the biocompatibility of the diamond.

The superlative electrical properties of boron-doped diamond (BDD) make it a desired material of choice for many neural interface applications. BDD can be either p-type [5-7] in character or quasi-metallic at high boron concentrations $\left(>10^{20} \mathrm{~cm}^{-3}\right)$ [8-10]. In its quasi-metallic state it is a desirable choice of material for bio-electrochemical electrodes due to its chemical inertness [11], high structural stability at desired current charge densities [12] and low background current [13, 14] compared to metal electrodes [15], both in vivo [16] and in vitro [17]. Moreover, the so-called electrochemical window of BDD electrodes, the voltage that can be applied before the onset of unwanted water redox reactions, is considerably higher than electrodes made for metals or other forms of carbon [18]. Boron doped nanocrystalline diamond (BNCD) has also been used for biosensing, with $\mathrm{H}$-terminated $\mathrm{BNCD}$ being used for the sensing of DNA $[19,20]$ and glucose [21], and O-terminated BNCD for sensing dopamine [22] and uric acid [23]. BNCD has been used material of choice for the fabrication of diamond microelectrode arrays (MEAs) [24-27], in particular Piret et al fabricated 3D-nanostructured BNCD MEAs with low level detection of neural activity, electrodes had a wide potential window and storage capacitance of $10 \mathrm{mC} \mathrm{cm}^{-2}$ [28]. BNCD is also a highly desirable material for neural prostheses and neural interfaces [29-31], as well as being used as a successful electrode material for neural stimulation [32].

For a material to be successful as a neural interface, it has to be both electrically active and biocompatible. The biocompatibility of un-doped CVD diamond, including NCD, has been investigated by cell viability and protein absorption methods, with most studies indicating that diamond is highly biocompatible [33-35]. BDD offers properties required for many cell interfacing applications, including the recording of action potentials from electrogenic cells [27]. To date, the biocompatibility of BNCD materials, where the boron concentration is as high as $10^{21} \mathrm{~cm}^{-3}$ has not been explored, that is the subject of the current paper. Of particular note is the use of vertically aligned CNTs (VACNTs) attached to substrates and subsequently overgrown with BNCD. This leads to a 3D structured BNCD material that can act as a scaffold for cellular attachment and outgrowth, and the biocompatibility of these novel 3D structures has also been explored. Planar thin films of BNCD can suffer from a relatively low double layer capacitance, and high impedance, reducing such electrodes performance for cell stimulation despite the attractive biocompatibility. 3D nanostructuring offers the prospect of overcoming this limitation, offering an innovative approach for electronic material-cellular interfacing.

The biocompatibility of diamond initiated a lot of work in which cells are grown on diamond, first reports of this occurred in 2004 by Specht et al [36]; where neurons were successfully grown on single crystal diamond, and since then, numerous reports have shown NCD positively supporting the adhesion and proliferation of many cell lines including osteoblasts [37-39], fibroblasts [40] and mesenchymal stem cells [41]. Patterned NCD films and nanodiamond layers have also been shown to direct neural adhesion and neurite outgrowth [34, 42, 43]. Here, the degree of human-neural stem cell (hNSC) adhesion and proliferation on diamond materials with these extreme levels of boron incorporation has been investigated via live cell staining using 5-chloromethylfluorescein diacetate (CMFDA); a live cell fluorescent marker, along with the use of a methylene blue assay. HNSCs have been used for this biocompatibility study due to their sensitivity and highly responsive behaviour to external stimuli; this is in contrast to robust cancerous cells.

\section{Methods}

\subsection{NCD and BNCD growth}

NCD films were grown on silicon substrates. Prior to growth the $\mathrm{Si}$ substrates were seeded with diamond nanoparticles by spin coating. high pressure high temperature diamond nanoparticles with diameter approximately $25 \mathrm{~nm}(0.1 \% \mathrm{wt} / \mathrm{wt}$, SYP GAF 0-0.05, Van Moppes) were dispersed in water $(0.1 \mathrm{wt} \%)$. The substrates were seeded by spin coating (2000 rpm for $20 \mathrm{~s}$ ) and dried in $\mathrm{N}_{2}$ gas (as previously reported by Girard et al) [44]. NCD was grown in an AX6500X microwave plasma enhanced chemical vapour deposition system (Seki Technotron Corp.). The growth was performed for $10 \mathrm{~h}$ using the following parameters: MW power of $2.6 \mathrm{~kW}$, pressure of $26 \mathrm{mbar}$, temperature of $650{ }^{\circ} \mathrm{C}, \mathrm{H}_{2}$ flow of $400 \mathrm{sccm}, \mathrm{CH}_{4}$ flow of $4 \mathrm{sccm}$. BNCD films were grown using these same growth parameters except trimethylborane (TMB, $\left.\mathrm{B}\left(\mathrm{CH}_{3}\right)_{3}\right)$ was added to the gas phase $(2.4 \% \mathrm{TMB}$ in $\mathrm{CH}_{4}$ ). Such conditions lead to BNCD with a boron concentration of ca. $2 \times 10^{21} \mathrm{~cm}^{-3}$, as described by Vanhove et al [13]. NCD and BNCD surfaces were oxygenated using an ozone treatment $(50 \mathrm{mbar})$ for two hours, at $200{ }^{\circ} \mathrm{C}$.

\subsection{Fabrication of BNCD coated CNTs}

VACNTs were deposited onto BNCD layers, prepared as described above. First, a $7 \mathrm{~nm}$ nickel layer was deposited on the diamond surface by e-beam evaporation and the sintered into $\sim 50 \mathrm{~nm}$ nanoparticles by heating at $700{ }^{\circ} \mathrm{C}$ for $3 \mathrm{~min}$ The samples were then transferred into a plasma enhanced CVD reactor, ('Black Magic' AIXTRON) whereby the sintered nickel particles act as the catalyst site in which VACNTs are grown. CNTs of differing length were deposited by varying growth times; $1 \mu \mathrm{m}$ and $2 \mu \mathrm{m}$ for growths of 5 and $11 \mathrm{~min}$ respectively. The VACNTs were then coated with NDs via an electrostatic grafting method described by Girard et al [44]. This step transforms the VACNTs into bundles of CNTs with densities of 9 and 4 bundles $\mu \mathrm{m}^{-2}$ for CNTs of length $1 \mu \mathrm{m}$ and $2 \mu \mathrm{m}$ respectively. Finally, BNCD was grown in a MWPECVD (AX6500X) reactor until a BNCD thin film coating of $\sim 50 \mathrm{~nm}$ was obtained upon the bundles. Boron incorporation was also optimised up to a boron concentration 
of $2 \times 10^{21} \mathrm{~cm}^{-3}$ such that the films became highly electrically conductive [4]. BNCD coated CNT surfaces were oxygenated using an ozone treatment as described above.

\subsection{Atomic force microscopy (AFM)}

AFM measurements were carried out using a Veeco Dimension $\mathrm{V}$ instrument with aluminum-coated silicon AFM probes (resonant frequency $190 \mathrm{kHz}$ ). The system was operated in tapping mode with a VT-103-3K acoustic/vibration isolation system and a VT-102 vibration isolation table at room temperature in air. AFM was performed on tissue culture polystyrene (TCPS) and on NCD and BNCD films. Scan sizes of $2 \mu \mathrm{m}$ for the TCPS and $5 \mu \mathrm{m}$ for the NCD and BNCD films were taken and an arithmetic average of the roughness profile $\left(R_{\mathrm{a}}\right)$ was calculated using Nanoscope Software 6.1.3. AFM Images were post processed with a median filter $(3 \times 3$ kernel) using MATLAB 2012a software to remove noise and measurement artifacts.

\subsection{Scanning electron microscopy (SEM).}

The BNCD covered CNT bundles were characterized using a Carl Zeiss XB1540 focused-ion-beam microscope with an accelerating voltage of $5 \mathrm{kV}$, these images were used to calculate bundle density.

\subsection{HNSCs isolation and culture.}

All procedures involving human tissue were carried out in accordance with the UK Human Tissue Act 2006. The hNSCs were isolated and expanded according to the protocol described previously by Sun et al [45]. Briefly, hindbrains from human embryos between 6-10 weeks old were collected through Human Developmental Biology Resource and dissected in cold Neurobasal medium (Gibco). After complete removal of the meninges and blood vessels, the tissue was chopped into smaller pieces and digested in Accutase (Gibco) solution at $37^{\circ} \mathrm{C}$ for $30 \mathrm{~min}$ with occasional trituration to obtain single cell suspension. Cells were then centrifuged and resuspended in growth medium composed of DMEM/F12 with Glutamax supplemented with $1 \%(\mathrm{v} / \mathrm{v})$ Penicillin/Streptomycin, $1 \%(\mathrm{v} / \mathrm{v}) 100 \times \mathrm{N} 2$ supplement, $2 \%(\mathrm{v} / \mathrm{v}) 50 \times \mathrm{B} 27$ supplement (all Gibco), $20 \mathrm{ng} \mathrm{ml}^{-1}$ human recombinant FGF2, $20 \mathrm{ng} \mathrm{ml}^{-1}$ human recombinant EGF (both Peprotech), $50 \mu \mathrm{g} \mathrm{ml}^{-1}$ BSA fraction $\mathrm{V}$ and $5 \mu \mathrm{g} \mathrm{ml}^{-1}$ Heparin (both Sigma). Cells were plated on polylysine/laminin $\left(10 \mu \mathrm{g} \mathrm{ml}^{-1}\right.$, Sigma)-coated dishes and grown for 7 days in vitro with the media changed every 2 days to remove any dead cells or debris. To eliminate neurons from the primary cultures and get a homogenous culture of neural stem cells, the cells were first transferred onto $0.1 \%(\mathrm{w} / \mathrm{v})$ bovine gelatine (Sigma)-coated dishes for 7 days to form neurospheres, which were then re-plated onto laminin-coated dishes for further expansion. For routine expansion and further experiments, cells were grown in growth media supplemented with laminin instead of coating the dishes. Passages up to 30 were used for all experiments.
2.5.1. Immunocytochemistry characterisation of hNSCs. For immunocytochemistry hNSCs were seeded either on polylysine/laminin coated glass cover slips or eight well imaging chamber slides (PAA). The cells were fixed with $4 \%(\mathrm{w} / \mathrm{v})$ paraformaldehyde (PFA) solution in $\mathrm{PBS}, \mathrm{pH}=7.4$ for $15 \mathrm{~min}$ at RT. After three rinses with PBS the cells were incubated with blocking solution composed of $10 \%$ (v/v) FBS, $3 \%(\mathrm{w} / \mathrm{v})$ BSA in PBS with $0.2 \%(\mathrm{v} / \mathrm{v})$ TritonX-100 for $1 \mathrm{~h}$ at RT to permeabilize cell membranes. Primary and secondary antibodies were diluted in blocking solution and the incubation times were overnight at $4{ }^{\circ} \mathrm{C}$ for primary, and $1 \mathrm{~h}$ at RT for secondary antibodies. Hoechst $33258\left(2 \mu \mathrm{g} \mathrm{ml}^{-1}\right)$ was added during secondary antibody incubation to counterstain cell nuclei. After final three washes in PBS the coverslips were mounted on slides with an aqueous based mounting medium (Hydromount, National Diagnostics). Primary and secondary antibodies used can be seen in the table 1 .

2.5.2. Induced hNSC differentiation. The controlled differentiation of hNSCs into astrocytes, oligodendrocytes and neurons has been adapted from Sun et al's protocol, as described in [45].

To induce astrocytic differentiation of hNSCs, the cells were cultured in media composed of DMEM/F12 medium, supplemented with $1 \%$ Penicillin/Streptomycin and $10 \%$ FBS for 2 weeks.

For neuronal differentiation hNSC were plated on laminin-coated plates in expansion medium without EGF supplement to induce differentiation. After 10 days in culture, FGF2 and heparin were also withdrawn from the media for another 4 days. Finally, neuron maturation was then induced by culturing in Neurobasal A medium supplemented with $1 \%$ Penicillin/Streptomycin, 1\% L-Glutamine, 2\% B27 and $10 \mathrm{ng} \mathrm{ml}^{-1}$ human recombinant $\beta \mathrm{NGF}$ with $10 \mathrm{ng} \mathrm{ml}^{-1}$ human recombinant BDNF (both Peprotech) for another 2 weeks.

For oligodendrocytic differentiation cells were plated on laminin coated plates. The next day, the medium was changed to DMEM/F12 supplemented with $1 \%$ Penicillin/Streptomycin, $1 \% \mathrm{~N} 2$ supplement, $10 \mathrm{nM}$ forskolin, $10 \mathrm{ng} \mathrm{ml}^{-1}$ FGF2 and $10 \mathrm{ng} \mathrm{ml}^{-1}$ PDGFaa (Peprotech) for 14 days. On day 15 the medium was switched to DMEM/F12 supplemented with $1 \%$ Penicillin/Streptomycin, 1\% N2 supplement, $30 \mathrm{ng} \mathrm{ml}^{-1}$ tri-iodothyronine (T3), $200 \mu \mathrm{M}$ ascorbic acid and $10 \mathrm{ng} \mathrm{ml}^{-1}$ PDGFaa. After another 7 days, PDGFaa was withdrawn from the culture media to allow maturation of cells. hNSC isolation, confirmation and differentiation potential extremely important as it allows for the development of cell based therapies to continue [46].

\subsection{Cell attachment and morphology assay}

Prior to cell seeding, substrates were treated with ozone in a vacuum chamber for $30 \mathrm{~min},\left(200{ }^{\circ} \mathrm{C}, 50 \mathrm{mbar}\right)$ in order to increase homogeneity of surface chemistry between samples. Substrates were then placed in 24 well tissue culture plates, sterilized for $30 \mathrm{~min}$ in $70 \%$ ethanol and washed three times in sterile distilled water. hNSCs were then plated at a density of $3 \times 10^{4}$ cells cm $\mathrm{cm}^{-2}$. Subconfluent hNSCs were labelled 
Table 1. Showing (a) primary antibodies and (b) secondary antibodies used throughout for specific hNSC, neuronal, oligodendrocytic and astrocytic fluorescent staining.

\begin{tabular}{|c|c|c|c|c|}
\hline $\begin{array}{l}\text { (a) } \\
\text { Name }\end{array}$ & Host & Company & Cat. number & \\
\hline GFAP & Rabbit & Millipore & AB 1540 & \\
\hline Nestin & Rabbit & Millipore & ABD69 & \\
\hline SOX2 & Rabbit & Millipore & AB5603 & \\
\hline$\beta$ III tubulin & Mouse & Promega & G712A & \\
\hline Vimentin & Mouse & Dako & M0725 & \\
\hline BLBP (FABP7) & Rabbit & Chemical signalling & D8N3N & \\
\hline MAP2 & Mouse & Life technologies & $13-1500$ & \\
\hline A2B5 & Mouse & R\&D systems & MAB1416 & \\
\hline \multicolumn{5}{|l|}{ (b) } \\
\hline Specificity & Host & Conjugated flurochrome & Company & Cat. number \\
\hline Anti rabbit & Donkey & Alexa568 & Molecular probes & A-110042 \\
\hline Anti mouse $\operatorname{IgG}$ & Donkey & Alexa488 & Molecular probes & A-21202 \\
\hline Anti mouse IgM & Goat & Alexa594 & Molecular probes & A-21044 \\
\hline
\end{tabular}

with the vital cytoplasmic dye, CMFDA (CMFDA, Cell Tracker, Invitrogen) in supplement-free culture media at $5 \mu \mathrm{M}$ final concentration for $30 \mathrm{~min}$. After the incubation, the cells were washed twice with phosphate buffer solution PBS, trypsinized and then plated on the different surfaces in the standard expansion media with laminin at a density of $3 \times 10^{4}$ cells $\mathrm{cm}^{-2}$. Cell attachment and morphology were assessed after $24 \mathrm{~h}$ using an Olympus IX70 inverted fluorescent microscope for one sample per substrate type. For each sample, six randomly selected areas were imaged using monochromatic Hamamatsu-OrcaR2 camera/HCImage software at $\times 10$ and $\times 40$ magnification.

For actin staining hNSCs were fixed after $48 \mathrm{~h}$ in vitro in $4 \%(\mathrm{w} / \mathrm{v})$ PFA in PBS (pH7.4) for $15 \mathrm{~min}$ and washed three times with PBS. Substrates were then incubated in blocking solution (10\% fetal bovine serum, $3 \%$ bovine serum albumin in PBS with $0.2 \%$ Triton $\times 100$ for permeablization) for $30 \mathrm{~min}$ at room temperature. Cells were stained with Phalloidin conjugated with Alexa Fluor ${ }^{\circledR} 488$ diluted in blocking buffer (Invitrogen, $5 \mathrm{U} \mathrm{ml}^{-1}$ ) together with Hoechst 33258 $\left(2 \mu \mathrm{g} \mathrm{ml}^{-1}\right)$ to counterstain nuclei for $1 \mathrm{~h}$ at room temperature. After three washes with PBS to remove excess dye the samples were imaged using an inverted microscope Olympus IX71 (Carl Zeiss, Jena, Germany) equipped with a Hamamatsu ORCA-ER digital camera (Hamamatsu Corp., Bridgewater, $\mathrm{NJ})$ and image processing was done using Image $\mathbf{J}$ and Fiji.

Culture confluence for both Phalloidin and CMFDA stained images were calculated in Matlab R2012b by applying an intensity threshold to the stained images and taking the average of the percentage area of each image above threshold (at least six images at varying magnification for one sample per substrate). Intensity thresholds were selected for each image by calculating the intensity level corresponding to the minimum value of the derivative of the intensity histogram of each image. This corresponded to the intensity boundary between the background and the cells. A 0.03 factor was added to this factor to improve mapping precision. Confluency was displayed using box plots, (figures 3(b) and 4(b)) Cell counts were obtained for the Hoechst stained images in Matlab R2012b by applying an intensity threshold to the stained images and then using a find circular Hough transform to find the number of labelled nuclei per image, which was then scaled up accordingly (at least six images at varying magnification for one sample per substrate).

\subsection{Methylene blue assay (MB)}

Cell viability of hNSCs was assessed by the methylene blue assay, which is a spectrophotochemically quantitative method for obtaining healthy cell counts [47]. Cell viability has been compared on NCD and BNCD using TCPS as the control throughout. hNSCs were plated on sterilized samples (three samples per substrate) in 48 well plates, at a density of $3 \times 10^{4}$ cells cm ${ }^{-2}$. After $48 \mathrm{~h}$ in culture cells were fixed in $4 \%$ $(\mathrm{w} / \mathrm{v})$ PFA in PBS (pH 7.4) for 15 min, washed once with PBS and incubated with $1 \%(\mathrm{w} / \mathrm{v})$ methylene blue in $0.01 \mathrm{M}$ borate buffer ( $\mathrm{pH}$ 8.5) solution at room temperature for $30 \mathrm{~min}$. Excess methylene blue in each well was washed off with $0.01 \mathrm{M}$ borate buffer. The samples were then transferred to new wells and the dye was eluted in $1: 1(\mathrm{v} / \mathrm{v})$ ethanol and $0.1 \mathrm{M}$ HCI. The plates were gently shaken, then $100 \mu \mathrm{l}$ of solution from each well were transferred to a 96 well plate and the absorbance was measured at $650 \mathrm{~nm}$ by a spectrophotometer (Revelation v4.21 Dynex Technologies). The absorbance measurements were then normalized to the surface area of the samples and compared to cells grown in standard TCPS.

\section{Results}

\subsection{Substrate characterization}

Figure 1 shows the surface topography of substrates used throughout this investigation. Figure 1(a) shows AFM images 
a)



$2 \mu \mathrm{m}$ square

$R_{a} 3 \pm 2 n m$

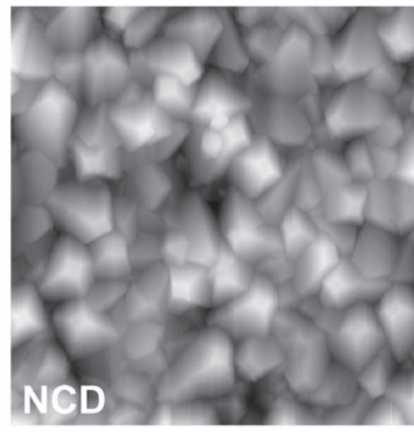

$5 \mu \mathrm{m}$ square $R_{a} 85 \pm 5 \mathrm{~nm}$
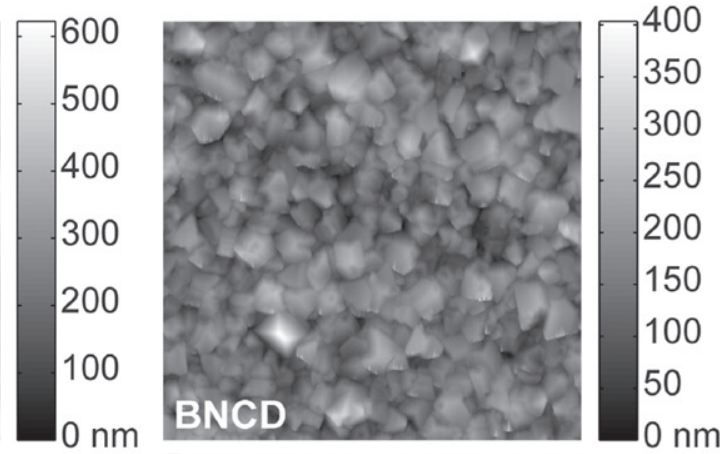

$5 \mu \mathrm{m}$ square $R_{a} 38 \pm 1 \mathrm{~nm}$

b)


Figure 1. (a) From left to right: $2 \mu \mathrm{m}$ square atomic force microscopy (AFM) scan of TCPS, and $5 \mu \mathrm{m}$ square scans for both NCD and BNCD films. Ra: average roughness. Approximate values given, averaged over three AFM scans. (b) Scanning electron microscopy (SEM) images of $1 \mu \mathrm{m}$ and $2 \mu \mathrm{m}$ CNT BCND nanostructures. $1 \mu \mathrm{m}$ CNT BNCD has ca. 9 bundles $\mu \mathrm{m}^{-2} .2 \mu \mathrm{m}$ CNT BNCD has 4 bundles $\mu \mathrm{m}^{-2}$. Scale bar $500 \mathrm{~nm} .5 \mathrm{kV}$ acceleration voltage.

of NCD, BNCD and TCPS substrates accompanied by the root mean square roughness for each substrate. The roughness of the NCD and BNCD is much greater than that of the TCPS, (being $85 \mathrm{~nm}$ and $38 \mathrm{~nm}$ respectively, versus $3 \mathrm{~nm}$ ). SEM has been used to show the structure of the BNCD CNT bundles, and figure 1(b) shows how the difference in length of the CNT alters the density of the bundles formed on the substrate. For CNTs of $1 \mu \mathrm{m}$ length, the concentration of bundles is $9 \mu \mathrm{m}^{-2}$ and for $2 \mu \mathrm{m}$ long CNTs this decreases to an average of ca. $4 \mu \mathrm{m}^{-2}$.

\subsection{Characterisation of the isolated hNSCs}

hNSCs have been isolated from human embryos aged between 6-10 weeks. Characterisation and confirmation of these cells has been summarized in figure 2, shows both the isolated hNSCs and the differentiation potential of cells from the same isolated batch. hNSCs have been successfully differentiated into neurons, astrocytes and oligodendrocytes, hereby proving the classification of the cells. Numerous NSC fluorescent markers have been used to clarify the identity of the isolated cells, including Nestin, GFAP, SOX2, Vimentin, TUBB3 and BLBP, and stained images expressing these proteins are shown in figure 2(a).

\subsection{Survival of $h N S C$ on $N C D, B N C D$ and $3 D$ nanostructured $B N C D$}

hNSCs were seeded onto ozone-treated NCD; BNCD; BNCD-coated CNTs of length $1 \mu \mathrm{m}$ and $2 \mu \mathrm{m}$; and TCPS, which was used as a control throughout. Cell attachment and morphology were assessed after $24 \mathrm{~h}$ in vitro using CMFDA, after $48 \mathrm{~h}$ via immunostaining using Phalloidin and Hoechst, and the methylene blue assay was used to evaluate cell viability on each substrate.

First of all the viability of hNSCs on planar substrates without CNT nanostructuring was investigated using immunohistochemical imaging. Fluorescent stained images in figure 3(a) show Phalloidin-labelled actin in green and nuclei stained by Hoechst in blue. The images are optically very similar and confluency percentage (figure 3(b)) shows that there is no statistical difference between the TCPS control, $\mathrm{NCD}$ and $\mathrm{BNCD}(37.6 \pm 4.8 \%, 37.2 \pm 6.4 \%, 37.1 \pm 8.7 \%$ respectively). Furthermore, no discernable differences were apparent in the morphology of adherent cells across each planar substrate. These similar confluency percentages and uniform cell morphology indicate that both NCD and BNCD are as good as the control for hNSC adhesion. Cell count data (figure 3(c)) for TCPS, NCD and BNCD substrates 
a) Characterisation of the isolated hNSCs:
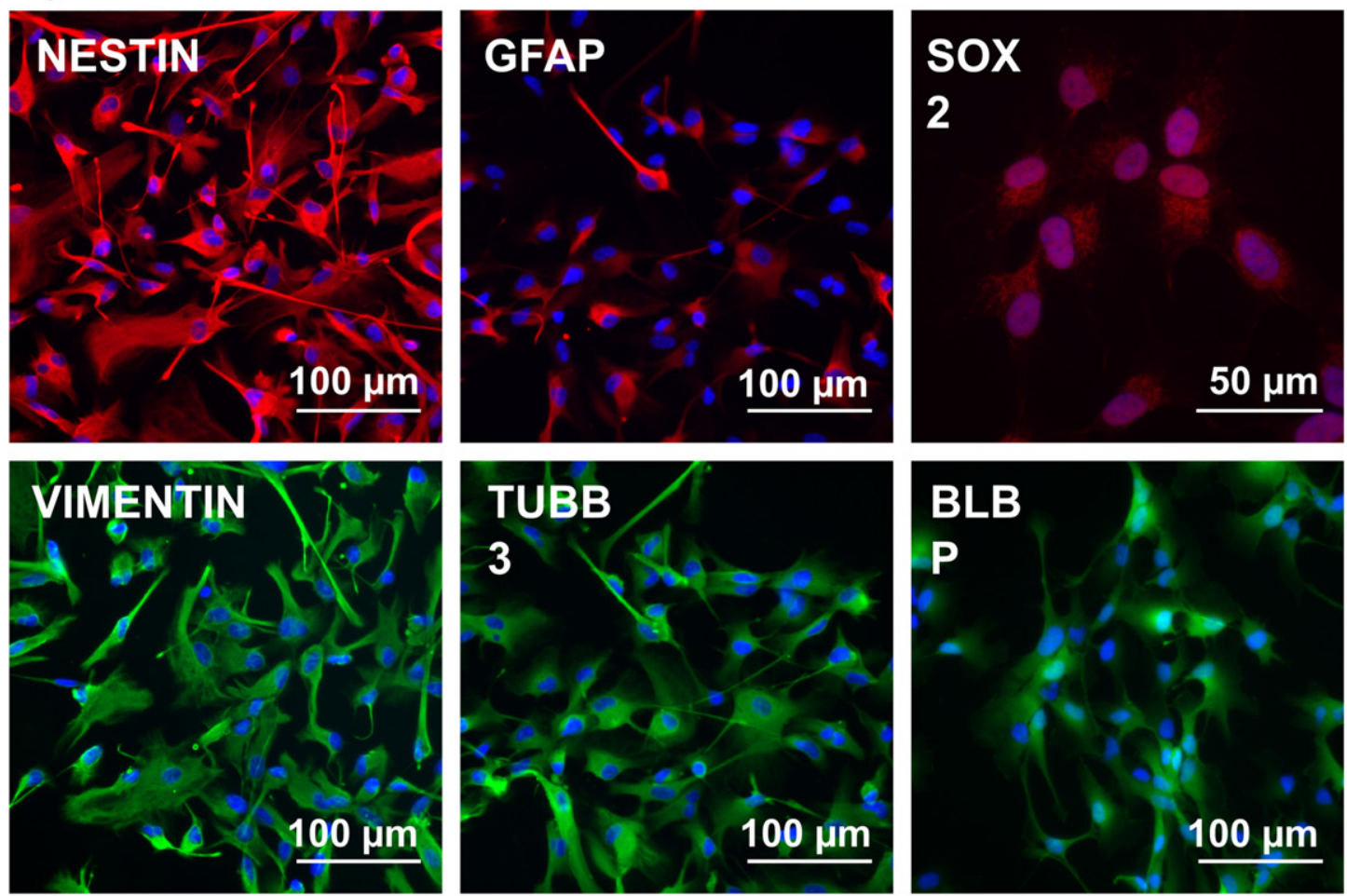

\section{b) Differentiation potential of hNSCs:}


Figure 2. Characterization of isolated hNSCs. (a) Expression of neural stem/progenitor cells markers in isolated hNSCs as detected by immunocytochemistry. All cells show strong expression of cytoskeletal proteins such as nestin, vimentin and $\beta$ III tubulin (TUBB3), while GFAP staining shows heterogeneity in the population with both low and high expressing cells. hNSCs also express SOX2 and radial glial marker BLBP. Nuclei are counterstained with Hoechst 33258 in blue. (b) Trilineage differentiation potential of hNSCs towards astrocytes, neurons and oligodendrocyte precursors. Top row: phase contrast images of hNSCs morphology after 2 weeks of differentiation. Bottom: expression of representative markers as detected by immunocytochemistry. 



Figure 3. (a) Fluorescently labelled images of hNSCs using Alexa Fluor $^{\circledR} 488$ Phalloidin (green) and Hoechst (blue) after $48 \mathrm{~h}$ in vitro on TCPS, NCD and BNCD. The scale bar is $100 \mu \mathrm{m}$ and $50 \mu \mathrm{m}$ for the $\times 10$ and $\times 40$ magnifications respectively. Panel (b) shows the confluency of each sample presented as a boxplot and mean of the data, panel (c) shows the boxplot and mean of cell concentration displayed as cells $\mathrm{cm}^{-2}$ (black dot: mean, vertical bars from left to right: minimum value, lower quartile, median, upper quartile, maximum value). Statistics performed using the threshold analysis on at least six images per sample.

also confirms the similarity of hNSC adherence with cell counts $\left(2.33 \times 10^{5}\right.$ cells cm ${ }^{-2}, 1.85 \times 10^{5}$ cells cm ${ }^{-2}$, and $2.09 \times 10^{5}$ cells cm $\mathrm{cm}^{-2}$ respectively).

Having established there to be no significant differences in hNSC viability between different substrate materials, cell viability was assessed for hNSC cultures upon CNT nanostructured substrates in comparison to planar substrates using live-cell staining and image analysis. CMFDA stained images in (figures 4(a) and (c)) show that the hNSCs have attached onto all surfaces, with a high confluence percentage and no indication of neurosphere formation, which can be indicative of hNSCs having poor adhesion to a biomaterial [48]. Further evidence of healthy attachment is demonstrated by the early formation of neurite processes after $24 \mathrm{~h}$ in vitro (figures 4(a) and (c) 2nd columns). The similar sub-confluent attachment (20-50\% mean confluence) of hNSCs over all substrates show that the cells are proliferating at similar rates. No significant change in confluence is observed between CNT BNCD substrates and the TCPSb control. In terms of the cells conforming to the BNCD-CNT structures, the grouping of cells is ascribed to fasciculation, rather than 3D structure obstruction, because the scale of fasciculation voids being considerably larger than the density of the 3D structures for both substrates (figure 4(b)), $1 \mu \mathrm{m}$ CNT BNCD: 9 bundles $\mu \mathrm{m}^{-2}, 2 \mu \mathrm{m}$ CNT BNCD: 4 bundles $\mu \mathrm{m}^{-2}$. No differences in morphology are observed among hNSCs grown on the different substrates.

The MB assay is a semi-quantitative method for indicating the relative number of healthy cells there are per substrate. Figure 5(a) MB assays reveal a slightly reduced cell count on NCD and BNCD samples in comparison to the TCPSa control. Error bars show standard deviation of the absorbance for each sample set (four substrates per sample), the larger error bars shown for NCD and BNCD result from using irregular sized substrates and so area calculations were not exact. Despite this, the cell counts for both NCD and BNCD are less than the TCPSa control. However, figure 5(b) shows very comparable cell counts between NCD and BNCD substrates, showing hNSCs to have a similar viability and propensity to attach and proliferate to NCD and BNCD surfaces. The nanostructured CNT BNCD samples showed significantly increased attachment of hNSCs in comparison to the TCPSb control, and moreover an increased cell count with increasing CNT length/reduced 3D structure density, (1 $\mu \mathrm{m}$ CNT 1.35 a.u., $2 \mu \mathrm{m}$ CNT 1.55 a.u., TCPSb 1.1 a.u.) was observed.

\section{Discussion}

Nanoscale topography has been shown to influence neuronal adhesion upon diamond [49], whereby surfaces with features of higher curvature promote neuronal adhesion. In NCD films curvature manifests in the size of grain boundaries, which in turn determines the roughness of substrates. The inclusion of boron in the diamond lattice during MWPECVD growth causes increased re-nucleation, resulting in smaller grains with higher curvature within BNCD materials, unlike intrinsic NCD materials which evolve larger grains during growth. In this case the reduced grain sizes of BNCD in comparison to NCD are still in excess of the dimensions that are conducive to nanotopographical enhancement of cellular adhesion (figure 1(a)). The similar values obtained from the MB assays for NCD and BNCD substrates (figures 5(a) and (b)) and the similar morphology of cells show the inclusion of boron within the diamond lattice not to be detrimental to hNSC adhesion, and that the reduced grain size of BNCD does not effect hNSC adhesion. The cell counts recorded here are comparable to murine NSCs grown on oxygenated ultrananocrystalline diamond [29]. Furthermore, the observation 



d)



Figure 4. Panels (a), (c) show live staining of hNSCs using CMFDA; a vital cytoplasmic dye after $24 \mathrm{~h}$ in vitro, on (a) TCPSa, NCD, BNCD and (c) TCPSb, $1 \mu \mathrm{m} \mathrm{CNT} \mathrm{BNCD} \mathrm{and} 2 \mu \mathrm{m}$ CNT BNCD. The scale bar is $100 \mu \mathrm{m}$, with the left hand column at $10 \times$ magnification and the right 40x. Panels (b), (d) show confluency of each sample presented as a boxplot and mean of the data (black dot: mean, vertical bars from left to right: minimum value, lower quartile, median, upper quartile, maximum value. Statistics performed using the threshold analysis on at least six images per sample).

a)

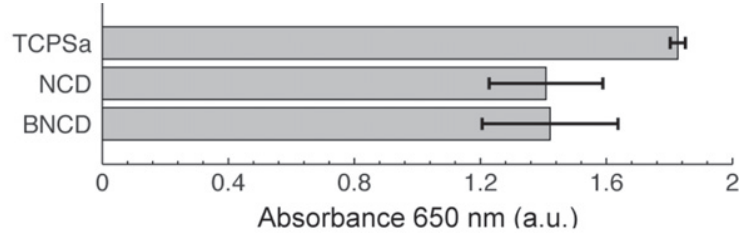

b)



Figure 5. Panels (a), (b) show the methylene blue assay indicative of vital cell count for each sample at an absorbance of $650 \mathrm{~nm}$.

Statistics performed using four substrates per sample set, the mean absorbance is plotted with error bars showing standard deviation.

of initial process extension of hNSCs on all substrates (figures 3(a) and 4(a), (c) 2nd columns), high confluency percentages (figures 2(b) and 3(b), (d) and similar cell counts for each substrate (figure 3(c)) are a promising indicator of healthy hNSCs and of subsequent neuronal network formation.
Upon 3D nanostructuring of BNCD substrates, the increased MB assay value of the CNT BNCD samples with respect to the TCPSb control substrate suggests nanostructuring enhances cell proliferation. Such an effect could be due to the increased surface area or enhanced local curvature of substrates. Given the monotonic increase in MB assay with respect to the length of CNTs, and the corresponding increase in sample surface area, this aspect of the substrate morphology could be ascribed to increasing cell proliferation. However, it has been shown [50] that cardiomyocytes cells preferentially sit atop of nanopillar features when spaced at a pitch of $2 \mu \mathrm{m}$ spacing. Herein, the spacing of the 3D structures is less than $2 \mu \mathrm{m}$ (figure 1(b)), hence, assuming hNSCs are of comparable size to cardiomyocyte cells, they are expected to be residing on top of the structures, thus surface area should not directly dictate cell count.

\section{Concluding remarks}

It has been found that the inclusion of boron in diamond has no observable effect of the proliferation and adherence of hNSCs. These results have been shown using image analysis 
of hNSCs grown on NCD and BNCD with TCPS as the control. Percentage confluency and cell count/MB data suggest that there is minimal difference between all three planar substrates. This significant result indicates that heavily BDD, which is required when diamond must be an electrical conductor, can be considered just as biocompatible as un-doped diamond. That this is even the case when such 'fragile' cells as hNSCs are used is encouraging for the use of, for example, 3D structured conductive diamond electrodes for several applications including deep brain and retinal implants. In addition to the assessment of BNCD versus NCD versus TCPS biocompatibility towards hNSCs, the impact of nanostructuring the BNCD using CNTs as scaffolds upon hNSC adherence and proliferation has been investigated. Herein it has been shown that increasing surface nanostructuring via CNT scaffolds has a positive effect on cell viability. Given the enhanced capacitance of such nanostructured electrodes, this result of increased cell viability of hNSCs on CNT-nanostructured BNCD is very promising for improving the performance of electrodes at the brain-machine interface, micro-electrode arrays and in lab-on-chip devices.

\section{Acknowledgments}

This work was performed as part of a EU F7 project 'NEUROCARE' (number 280433-2) and was partially supported by the UKs Engineering and Physical Sciences Research Council (EPSRC, EP/F026110/1).

\section{References}

[1] May P W 2000 Diamond thin films: a 21st-century material Phil. Trans. R. Soc. A 358 473-95

[2] Fries M, D and Vohra Y, K 2004 Properties of nanocrystalline diamond thin films grown by MPCVD for biomedical implant purposes Diam. Relat. Mater. 13 1740-3

[3] Williams O, A and Nesladek M 2006 Growth and properties of nanocrystalline diamond films Phys. Status Solidi a $\mathbf{1 3}$ 3375-86

[4] Hebert C, Mazellier J P, Scorsone E, Mermoux M and Bergonzo P 2014 Boosting the electrochemical properties of diamond electrodes using carbon nanotube scaffolds Carbon 71 27-33

[5] Fontaine F, Saguy C U and Philosoph B 1996 Boron implantation/in situ annealing procedure for optimal p-type properties of diamond Appl. Phys. Lett. 682264

[6] Braunstein G and Kalish R 1983 Effective p-type doping of diamond by boron ion implantation J. Appl. Phys. 542106

[7] Visser E P, Bauhuis G J, Janssen G, Vollenberg W, van Enckevort J P and Giling L J 1999 Electrical conduction in homoepitacial, boron-doped diamond films $J$. Phys.: Condens. Matter 4 7365-76

[8] Yokoya T, Nakamura T, Matsushita T, Muro T, Takano Y, Nagao M, Takenouchi T, Kawarada H and Oguchi T 2005 Origin of the metallic properties of heavily boron-doped superconducting diamond Nature 438 647-50

[9] Bustarret E, Achatz P, Sacépé B, Chapelier C, Marcenat C, Ortéga L and Klein T 2008 Metal-to-insulator transition and superconductivity in boron-doped diamond Phil. Trans. $R$. Soc. A 366 267-79
[10] Klein T, Achatz P, Kačmarčik J and Marcenat C 2007 Metalto-insulator transition and superconductivity in boron-doped diamond Phys. Rev. B 75165313

[11] Hupert M, Muck A, Wang J, Stotter J, Cvackova Z, Haymond S, Show Y and Swain G M 2003 Conductive diamond thin-films in electrochemistry Diam. Relat. Mater. 12 1940-9

[12] Chaplin B P, Hubler D K and Farrell J 2013 Understanding anodic wear at boron doped diamond film electrodes Electrochim. Acta 89 122-31

[13] Vanhove E, De Sanoit J and Mailley P 2009 High reactivity and stability of diamond electrodes: the influence of the B-doping concentration Phys. Status Solidi A 206 2063-9

[14] Wei J J, Li C M, Gao X H, Hei L F and Lvun F X 2012 The influence of boron doping level on quality and stability of diamond film on Ti substrate Appl. Surf. Sci. 258 6909-13

[15] Fujishima A 2005 Diamond Electrochemistry (Amsterdam: Elsevier)

[16] Suzuki A, Ivandini T A, Yoshimi K, Fujishima A, Oyama G, Nakazato T, Hattori N, Kitazawa S and Einaga Y 2007 Fabrication, characterisation and application of boron-doped diamond electrodes for in vivo dopamine detection Anal. Chem. 79 8608-15

[17] Park J, Quaiserová-Mocko V, Patel B A, Novotný M, Liu A, Bian X, Galligan J J and Swain G M 2008 Diamond microelectrodes for in vitro electroanalytical measurements: current status and remaining challenges Analyst 133 17-24

[18] Panizza M and Cerisola G 2005 Application of diamond electrodes to electrochemical processes Electrochim. Acta. 52 191-9

[19] Wenmackers S, Vermeeren V, vandeVen M, Ameloot M, Bijnens N, Haenen K, Michiels L and Wagner P 2009 Diamond-based DNA sensors: surface functionalization and read-out strategoes Phys. Status Soldi a 206 391-408

[20] Vermeeren V, Wenmackers S, Wagner P and Michiels L 2009 DNA sensors with diamond as a promising alternative transducer material Sensors 9 5600-36

[21] Lee J and Park S M 2005 Direct electrochemical assay of glucose using boron-doped diamond electrodes Anal. Chim. Acta $54527-32$

[22] Popa E 1999 Selective electrochemical detection of dopamine in the presence of ascorbic acid at anodized diamond thin film electrodes Electrochem. Solid-State Lett. 249

[23] Popa E, Kubota Y, Tryk D A and Fujishima A 2000 Selective voltammetric and amperometric detection of uric acid with oxidized diamond film electrodes Anal. Chem. 72 1724-7

[24] Bonnauron M, Saada S, Rousseau L, Lissorgues G, Mer C and Bergonzo P 2008 Diam. Relat. Mater. 17 1399-404

[25] Bergonzo P et al 2011 3D shaped mechanically flexible diamond microelectrode arrays for eye implant applications: the MEDINAS project IRBM 32 91-4

[26] Hebert C et al 2014 Boron doped diamond biotechnology: from sensors to neurointerfaces Faraday Discuss. 172 47-59

[27] Maybeck V, Edgington R, Bongrain A, Welch J O, Scorsone E, Bergonzo P, Jackman R B and Offenhäusser A 2014 Boron-doped nanocrystalline diamond microelectrode arrays monitor cardiac action potentials Adv. Healthc. Mater. 3 283-9

[28] Piret G et al 2015 3D-nanostructured boron-doped diamond for microelectrode array neural interfacing Biomaterials 53 $173-83$

[29] Chen Y C, Lee D C, Hsiao C Y, Chung Y F, Chen H C, Palathinkal T J, Pong W F, Tai N H, Lin I N and Chiu I M 2009 The effect of ultra-nanocrystalline diamond films on the proliferation and differentiation of neural stem cells Biomaterials 30 3428-35

[30] Chen Y C et al 2010 Induction and regulation of differentiation in neural stem cells on ultra-nanocrystalline diamond films Biomaterials 31 5575-87 
[31] Ariano P, Budnyk O, Dalmazzo S, Lovisolo D, Manfredotti C, Rivolo P and Vittone E 2009 On diamond surface properties and interactions with neurons Eur. Phys. J. E 30 149-56

[32] Garrett D J, Ganesan K, Stacey A, Fox K, Meffin H and Prawer S 2012 Ultra-nanocrystalline diamond electrodes: optimization towards neural stimulation applications J. Neural Eng. 9016002

[33] Amaral M, Dias A G, Gomes P S, Lopes M A, Silva R F, Santos J D and Fernandes M H 2008 Nanocrystalline diamond: in vitro biocompatibility assessment by MG63 and human bone marrow cells cultures J. Biomed. Mater. Res. A 87 91-9

[34] May P W, Regan E M, Taylor A, Uney J, Dick A D and Mcgeehan J 2012 Spatially controlling neuronal adhesion on CVD diamond Diam. Relat. Mater. 23 1-5

[35] Tang L, Tsai C, Gerberich W W, Kruckeberg L and Kania D R 1995 Biocompatability of chemical-vapour-deposited diamond J. Neurosci. Methods 16 483-8

[36] Specht C, Williams O, Jackman R and Schoepfer R 2004 Ordered growth of neurons on diamond Biomaterials $\mathbf{2 5}$ 4073-8

[37] Kalbacova M, Rezek B, Baresova V, Wolf-Brandstetter C and Kromka A 2009 Nanoscale topography of nanocrystalline diamonds promotes differentiation of osteoblasts Acta Biomater. 5 3076-85

[38] Kalbacova M, Broz A, Babchenko O and Kromka A 2009 Study on cellular adhesion of human osteoblasts on nano-structured diamond films Phys. Status Solidi b 246 2774-7

[39] Yang L, Li Y, Sheldon B W and Webster T J 2011 Altering surface energy of nanocrystalline diamond to control osteoblast responses Lab Chip 22205

[40] Amaral M, Gomes P S, Lopes M A, Santos J D, Silva R F and Fernandes M H 2009 Cytotoxicity evaluation of nanocrystalline diamond coatings by fibroblast cell cultures Acta Biomater. 5 755-63

[41] Clem W C, Chowdhury S, Catledge S A and Weimer J J 2008 Mesenchymal stem cell interaction with ultra-smooth nanostructured diamond for wear-resistant orthopaedic implants Biomaterials 29 3461-8

[42] Regan E M, Taylor A, Uney J B, Dick A D, May P W and McGeehan J 2011 Spatially controlling neuronal adhesion and inflammatory reactions on implantable diamond IEEE JETCAS 1 557-65

[43] Thalhammer A, Edgington R J, Cingolani L A, Schoepfer R and Jackman R B 2012 The use of nanodiamond monolayer coatings to promote the formation of functional neuronal networks Biomaterials 3 2097-104

[44] Girard H A, Perruchas S and Gesset C 2009 Electrostatic grafting of diamond nanoparticles: a versatile route to nanocrystalline diamond thin films ACS Appl. Mater. Interfaces 1 2738-46

[45] Sun Y, Pollard S, Conti L, Toselli M, Biella G, Parkin G, Willatt L, Falk A, Cattaneo E and Smith A 2008 Long-term tripotent differentiation capacity of human neural stem (NS) cells in adherent culture Mol. Cell. Neurosci. 38 245-58

[46] New S E P, Alvarez-Gonzalez C, Vagaska C, Gomez S G, Bulstrode N W, Madrigal A and Ferretti P 2015 A matter of identity-phenotype and differentation potential of human somatic stem cells Stem Cell Res. 15 1-13

[47] Lagneau A, Martin M, Martin F and Michel M F 1977 Cytotoxic effect of peritoneal macrophages on an intestinal carcinoma in rats. Demonstration of a new cytotoxicity test C. R. Seances Soc. Biol. Fil. 171 90-3

[48] Pacey L, Stead S, Gleave J, Tomczyk K and Doering L 2006 Neural stem cell culture: neurosphere generation, microscopical analysis and cryopreservation Protocol Exchange doi: $10.1038 /$ nprot.2006.215

[49] Edgington R J, Thalhammer A, Welch J O, Bongrain A, Bergonzo P, Scorsone E, Jackman R B and Schoepfer R 2013 Patterned neuronal networks using nanodiamonds and the effect of varying nanodiamond properties on neuronal adhesion and outgrowth J. Neural Eng. 10056022

[50] Santoro F, Schnitker J, Panaitov G and Offenhäusser A 2013 On chip guidance and recording of cardiomyocytes with 3D mushroom-shaped electrodes Nano Lett. 13 5379-84 